\title{
Non-commutative martingale VMO-spaces
}

\author{
by \\ NARCisse RandRianantoanina (Oxford, OH)
}

\begin{abstract}
We study Banach space properties of non-commutative martingale VMOspaces associated with general von Neumann algebras. More precisely, we obtain a version of the classical Kadets-Pełczyński dichotomy theorem for subspaces of non-commutative martingale VMO-spaces. As application we prove that if $\mathcal{M}$ is hyperfinite then the noncommutative martingale VMO-space associated with a filtration of finite-dimensional von Neumannn subalgebras of $\mathcal{M}$ has property (u).
\end{abstract}

1. Introduction. The space of functions of bounded mean oscillation generally referred to as BMO-space has been instrumental in several aspects of analysis. Its martingale version plays an equally important role in probability.

In this paper, we analyze subspaces of BMO-spaces related to non-commutative martingales. Our main motivation comes primarily from a paper by Müller and Schechtman [14] who studied structural properties of closed subspaces of dyadic martingale VMO (vanishing mean oscillation) as Banach spaces. More precisely, they provided, among other things, a version of the classical Kadets-Pełczyński dichotomy theorem for closed subspaces of dyadic martingale VMO-spaces. In order to explain the details, we first recall the celebrated Kadets-Pełczyński dichotomy theorem, which states that every closed subspace of $L^{p}(0,1), 2<p<\infty$, either is isomorphic to a Hilbert space or contains a subspace which is isomorphic to $l^{p}$. This dichotomy plays a crucial role in the development of $L^{p}$-spaces and the theory of function spaces in general. Non-commutative analogues of the Kadets-Pełczyński dichotomy has been considered by several authors with the most general result obtained by Raynaud and Xu (see [21]) in the context of Haagerup $L^{p}$-spaces when $2 \leq p<\infty$. Clearly, the dichotomy does not extend to closed subspaces of $L^{\infty}(0,1)$ or any $C(K)$-spaces in general. As a substitute, the following result was obtained by Müller and Schechtman:

2000 Mathematics Subject Classification: Primary 46B03, 46L52; Secondary 46B10.

Key words and phrases: non-commutative $L^{p}$-spaces, non-commutative martingale BMOspaces.

Research partially supported by NSF grant DMS-0456781. 
Theorem 1.1 ([14]). Let $X$ be a subspace of the dyadic VMO. Then either $X$ is isomorphic to a Hilbert space or $X$ contains a subspace isomorphic to $c_{0}$.

The result of Raynaud and Xu naturally leads to the question whether Theorem 1.1 generalizes to non-commutative martingale VMO. Our main result (Theorem 3.2) can be viewed as a characterization of subspaces of non-commutative VMO that contain isomorphic copies of $c_{0}$, which for the hyperfinite case (Theorem 3.4) becomes an exact non-commutative analogue of Theorem 1.1. As application, we deduce that as Banach space, non-commutative VMO has the so called property $(u)$.

The paper is organized as follows: in Section 2, we recall the construction of Haagerup $L^{p}$-spaces associated with general von Neumannn algebras, review the general setup for non-commutative martingales, and introduce non-commutative VMO-spaces. In Section 3, we formulate the appropriate analogue of the Kadets-Pełczyński dichotomy theorem for VMO-spaces. In the last section, we discuss applications of our main result to Banach space structures of both VMO and BMO.

2. Notation and preliminary definitions. We use standard notation in operator algebras and Banach space theory. We refer to [11, 23] for background on von Neumannn algebra theory and to [3, 12] for unexplained terminology from Banach space theory. Throughout, we assume that $\mathcal{M}$ is a $\sigma$-finite von Neumannn algebra acting on a Hilbert space $H$, and $\varphi$ is a distinguished normal faithful state on $\mathcal{M}$. We denote by $\sigma_{t}=\sigma_{t}^{\varphi}$ the one-parameter modular automorphism group on $\mathcal{M}$ associated with $\varphi$. The Haagepup $L^{p}$-spaces associated with $(\mathcal{M}, \varphi)$ are defined from the crossproduct $\mathcal{R}=\mathcal{M} \rtimes_{\sigma_{t}} \mathbb{R}$. We recall that $\mathcal{R}$ is the von Neumannn subalgebra of $\mathcal{B}\left(L^{2}(\mathbb{R}, H)\right)$ generated by the operators $\pi(x), x \in \mathcal{M}$, and $\lambda(s), s \in \mathbb{R}$, where

$$
\pi(x)(\xi(t))=\sigma_{-t}^{\varphi}(x)(\xi(t)) \quad \text { and } \quad \lambda(s)(\xi(t))=\xi(t-s)
$$

for $t \in \mathbb{R}$ and $\xi \in L^{2}(\mathbb{R}, H)$. If $W(s)$ is the unitary operator on $L^{2}(\mathbb{R}, H)$ defined by

$$
W(s)(\xi(t))=e^{-i s t} \xi(t),
$$

then the dual action $\theta$ on $\mathcal{R}$ is given by

$$
\theta_{s}(x)=W(s) x W(s)^{*}, \quad x \in \mathcal{R} .
$$

The von Neumannn algebra $\mathcal{M}$ can be identified with the subalgebra of $\mathcal{R}$

$$
\pi(\mathcal{M})=\left\{x \in \mathcal{R}: \theta_{s}(x)=x \text { for all } s \in \mathbb{R}\right\} .
$$

Moreover, it is known that $\mathcal{R}$ is a semifinite von Neumannn algebra and admits a canonical normal faithful semifinite trace $\tau$ satisfying

$$
\tau \circ \theta_{s}=e^{-s} \tau, \quad s \in \mathbb{R}, x \in \mathcal{R} .
$$


For $1 \leq p \leq \infty$, the Haagerup $L^{p}$-space associated with $\mathcal{M}$, denoted by $L^{p}(\mathcal{M})$, is defined as the space of $\tau$-measurable operators $x$ affiliated with $\mathcal{R}$ such that for all $s \in \mathbb{R}$,

$$
\theta_{s}(x)=e^{-s / p} x .
$$

This is clearly a closed self-adjoint linear subspace of the space of $\tau$-measurable operators affiliated with $\mathcal{R}$, and $L^{\infty}(\mathcal{M})$ coincides with $\mathcal{M}$. Moreover, there is a canonical isomorphism between $L^{1}(\mathcal{M})$ and the predual $\mathcal{M}_{*}$ of $\mathcal{M}$ which we now describe:

Every normal semifinite faithful weight $\psi \in\left(\mathcal{M}_{*}\right)_{+}$is given by a density $h_{\psi} \in L^{1}(\mathcal{M})_{+}$satisfying

$$
\tau\left(h_{\psi} x\right)=\int_{\mathbb{R}} \psi\left(\theta_{s}(x)\right) d s
$$

for all $x \in \mathcal{R}_{+}$. Using polar decomposition of an arbitrary element $\psi \in \mathcal{M}_{*}$, this correspondence between $\left(\mathcal{M}_{*}\right)_{+}$and $L^{1}(\mathcal{M})_{+}$extends to a bijection between $\mathcal{M}_{*}$ and $L^{1}(\mathcal{M})$. Indeed, if $\psi \in \mathcal{M}_{*}$, then $\psi=u|\psi|$, where $u \in \mathcal{M}$ and $|\psi|$ is the modulus of $\psi$. The corresponding $h_{\psi} \in L^{1}(\mathcal{M})$ then admits the polar decomposition

$$
h_{\psi}=u\left|h_{\psi}\right|=u h_{|\psi|} .
$$

The norm on $L^{1}(\mathcal{M})$ is defined by setting

$$
\left\|h_{\psi}\right\|_{1}=|\psi|(\mathbf{1})=\|\psi\|_{\mathcal{M}_{*}}, \quad \psi \in \mathcal{M}_{*} .
$$

With this norm, $L^{1}(\mathcal{M})$ and $\mathcal{M}_{*}$ are isometric. Furthermore, one can define a distinguished positive linear functional $\operatorname{tr}$ on $L^{1}(\mathcal{M})$ called trace by

$$
\operatorname{tr}\left(h_{\psi}\right)=\psi(\mathbf{1}), \quad \psi \in \mathcal{M}_{*} .
$$

Given $1 \leq p<\infty$ and $x \in L^{p}(\mathcal{M})$, we have $|x|^{p} \in L^{1}(\mathcal{M})$. Define

$$
\|x\|_{p}:=\left\||x|^{p}\right\|_{1}^{1 / p}=\left(\operatorname{tr}\left(|x|^{p}\right)\right)^{1 / p} .
$$

Equipped with $\|\cdot\|_{p}$, the space $L^{p}(\mathcal{M})$ is a Banach space.

Throughout, $D$ denotes the Radon-Nikodym derivative (with respect to $\tau$ ) of the dual weight $\widetilde{\varphi}$ of the distinguished state $\varphi$. The state $\varphi$ can be recovered from tr by the identity

$$
\varphi(x)=\operatorname{tr}(D x), \quad x \in \mathcal{M} .
$$

If the von Neumannn algebra $\mathcal{M}$ is semifinite equipped with a faithful normal semifinite trace then the Haagerup $L^{p}$-spaces reduce to the usual noncommutative $L^{p}$-spaces constructed from the theory of non-commutative integration as described in [16]. The reader is referred to [7, 24] for full details of Haagerup's theory.

Let us now recall the general setup for non-commutative martingales. The reader is referred to $[4,2,5]$ for the classical (commutative) martingale 
theory. Let $\left(\mathcal{M}_{n}\right)_{n \geq 1}$ be an increasing sequence of von Neumannn subalgebras of $\mathcal{M}$ such that the union of the $\mathcal{M}_{n}$ 's is weak*-dense in $\mathcal{M}$ and $\sigma_{t}\left(\mathcal{M}_{n}\right) \subset \mathcal{M}_{n}$ for all $t \in \mathbb{R}$ and all $n \in \mathbb{N}$. We further assume that if $\left(p_{n}\right)_{n \geq 1}$ is the increasing sequence of projections in $\mathcal{M}$ consisting of the units of $\mathcal{M}_{n}$ 's then $\sigma_{t}\left(p_{n}\right)=p_{n}$ for all $t \in \mathbb{R}$ and all $n \in \mathbb{N}$.

For each $n \geq 1$, it follows from [22] that there exists a normal conditional expectation $\widetilde{\mathcal{E}}_{n}: p_{n} \mathcal{M} p_{n} \rightarrow \mathcal{M}_{n}$ satisfying

$$
\widetilde{\mathcal{E}}_{n}\left(\sigma_{t}(x)\right)=\sigma_{t}\left(\widetilde{\mathcal{E}}_{n}(x)\right)
$$

for all $x \in p_{n} \mathcal{M} p_{n}$ and all $t \in \mathbb{R}$. We now define $\mathcal{E}_{n}: \mathcal{M} \rightarrow \mathcal{M}_{n}$ by setting, for $x \in \mathcal{M}$,

$$
\mathcal{E}_{n}(x)=\widetilde{\mathcal{E}}_{n}\left(p_{n} x p_{n}\right) .
$$

It is clear that for every $m$ and $n$ in $\mathbb{N}, \mathcal{E}_{m} \mathcal{E}_{n}=\mathcal{E}_{n} \mathcal{E}_{m}=\mathcal{E}_{\min (n, m)}$. Moreover, for $1 \leq p<\infty$, the conditional expectation $\mathcal{E}_{n}$ extends in a natural way to a contractive projection from $L^{p}(\mathcal{M})$ onto $L^{p}\left(\mathcal{M}_{n}\right)$ (we refer to [10, Lemma 2.2] for details).

Definition 2.1. For $1 \leq p \leq \infty$, a non-commutative martingale in $L^{p}(\mathcal{M})$ with respect to the filtration $\left(\mathcal{M}_{n}\right)_{n \geq 1}$ is a sequence $x=\left(x_{n}\right)_{n \geq 1}$ in $L^{p}(\mathcal{M})$ satisfying

$$
\mathcal{E}_{n}\left(x_{n+1}\right)=x_{n} \quad \text { for all } n \geq 1 .
$$

For such a martingale $x \subset L^{p}(\mathcal{M})(1 \leq p \leq \infty)$, we set

$$
\|x\|_{p}:=\sup _{n \geq 1}\left\|x_{n}\right\|_{p} .
$$

If $\|x\|_{p}<\infty$, then $x$ is called a bounded $L^{p}$-martingale. The difference sequence $d x=\left\{d_{n}(x)\right\}_{n \geq 1}$ of a martingale $x=\left(x_{n}\right)_{n \geq 1}$ is defined by

$$
d_{n}(x)=x_{n}-x_{n-1}, \quad n \geq 1,
$$

with the usual convention that $x_{0}=0$.

We note that for $1<p<\infty$, every bounded $L^{p}$-martingale is of the form $\left(\mathcal{E}_{n}\left(x_{\infty}\right)\right)_{n \geq 1}$ for some $x_{\infty} \in L^{p}(\mathcal{M})$. We often identify a martingale with its final value whenever the latter exists. For some concrete natural examples of non-commutative martingales, we refer to [20] and the survey paper [26].

We will now describe square functions of non-commutative martingales and non-commutative martingale Hardy spaces. Our main references for classical martingale Hardy spaces are the monographs [13, 25]. Following Pisier and $\mathrm{Xu}[20]$, we will consider the following row and column versions of square functions. For a martingale $x=\left(x_{n}\right)_{n=1}^{\infty}$ and $N \geq 1$, set

$$
S_{\mathrm{C}, N}(x)=\left(\sum_{j=1}^{N}\left|d_{j}(x)\right|^{2}\right)^{1 / 2} \quad \text { and } \quad S_{\mathrm{R}, N}(x)=\left(\sum_{j=1}^{N}\left|d_{j}\left(x^{*}\right)\right|^{2}\right)^{1 / 2} \text {. }
$$


For $1 \leq p \leq \infty$ and any finite sequence $a=\left(a_{n}\right)_{n \geq 1}$ in $L^{p}(\mathcal{M})$, set

$$
\begin{aligned}
& \|a\|_{L^{p}\left(\mathcal{M} ; l_{\mathrm{C}}^{2}\right)}=\left\|\left(\sum_{n \geq 1}\left|a_{n}\right|^{2}\right)^{1 / 2}\right\|_{L^{p}(\mathcal{M}, \tau)}, \\
& \|a\|_{L^{p}\left(\mathcal{M} ; l_{\mathrm{R}}^{2}\right)}=\left\|\left(\sum_{n \geq 1}\left|a_{n}^{*}\right|^{2}\right)^{1 / 2}\right\|_{L^{p}(\mathcal{M}, \tau)} .
\end{aligned}
$$

The difference sequence $d x$ belongs to $L^{p}\left(\mathcal{M} ; l_{\mathrm{C}}^{2}\right)\left(\right.$ respectively, $\left.L^{p}\left(\mathcal{M} ; l_{\mathrm{R}}^{2}\right)\right)$ if and only if the sequence $\left(S_{\mathrm{C}, n}(x)\right)_{n=1}^{\infty}$ (respectively, $\left.\left(S_{\mathrm{R}, n}(x)\right)_{n=1}^{\infty}\right)$ is bounded in $L^{p}(\mathcal{M})$. In this case, the limits

$$
S_{\mathrm{C}}(x)=\left(\sum_{k=1}^{\infty}\left|d_{k}(x)\right|^{2}\right)^{1 / 2} \quad \text { and } \quad S_{\mathrm{R}}(x)=\left(\sum_{k=1}^{\infty}\left|d_{k}(x)^{*}\right|^{2}\right)^{1 / 2}
$$

are elements of $L^{p}(\mathcal{M})$. These two versions of square functions are crucial for the definition of non-commutative (martingale) Hardy spaces which we now describe for $p=1$ (we refer to [20] for the other values of $p$ ). The space $\mathcal{H}_{\mathrm{C}}^{1}(\mathcal{M})$ (respectively, $\mathcal{H}_{\mathrm{R}}^{1}(\mathcal{M})$ ) is defined as the set of all $L^{1}$-martingales $x$ with respect to the filtration $\left(\mathcal{M}_{n}\right)_{n \geq 1}$ for which $d x \in L^{1}\left(\mathcal{M} ; l_{\mathrm{C}}^{2}\right)$ (respectively, $\left.L^{1}\left(\mathcal{M} ; l_{\mathrm{R}}^{2}\right)\right)$. For such $x$, we set

$$
\|x\|_{\mathcal{H}_{\mathrm{C}}^{1}(\mathcal{M})}=\left\|S_{\mathrm{C}}(x)\right\|_{1} \quad \text { and } \quad\|x\|_{\mathcal{H}_{\mathrm{R}}^{1}(\mathcal{M})}=\left\|S_{\mathrm{R}}(x)\right\|_{1} .
$$

Equipped with the above norms, $\mathcal{H}_{\mathrm{C}}^{1}(\mathcal{M})$ and $\mathcal{H}_{\mathrm{R}}^{1}(\mathcal{M})$ are Banach spaces. The non-commutative martingale Hardy space $\mathcal{H}^{1}(\mathcal{M})$ of non-commutative martingales is defined as

$$
\mathcal{H}^{1}(\mathcal{M})=\mathcal{H}_{\mathrm{C}}^{1}(\mathcal{M})+\mathcal{H}_{\mathrm{R}}^{1}(\mathcal{M})
$$

equipped with the usual norm of sum of two Banach spaces:

$$
\|x\|_{\mathcal{H}^{1}(\mathcal{M})}=\inf \left\{\|y\|_{\mathcal{H}_{\mathrm{C}}^{1}(\mathcal{M})}+\|z\|_{\mathcal{H}_{\mathrm{R}}^{1}(\mathcal{M})}\right\}
$$

where the infimum is taken over all $y$ and $z$ with $x=y+z, y \in \mathcal{H}_{\mathrm{C}}^{1}(\mathcal{M})$ and $z \in \mathcal{H}_{\mathrm{R}}^{1}(\mathcal{M})$.

The dual of $\mathcal{H}^{1}(\mathcal{M})$ can be identified (up to a constant $\sqrt{2}$ ) to a noncommutative analogue of martingale BMO which is the main object of this paper. BMO-spaces associated with non-commutative martingales were introduced by Pisier and $\mathrm{Xu}$ in [20] for the finite case and [10] for the general case. It was proved among other results that Fefferman's classical $H^{1}$-BMO duality extends to this setting. In order to define such BMO-spaces for this context, we consider first what is known as the column BMO by setting

$$
\operatorname{BMO}_{\mathrm{C}}(\mathcal{M}):=\left\{x=\left(x_{n}\right): \sup _{m} \sup _{n \leq m}\left\|\mathcal{E}_{n}\left|x_{m}-x_{n-1}\right|^{2}\right\|_{\infty}<\infty\right\} .
$$


Then $\mathrm{BMO}_{\mathrm{C}}(\mathcal{M})$ becomes a Banach space when equipped with the norm

$$
\|x\|_{\mathrm{BMO}_{\mathrm{C}}}=\left(\sup _{m \geq 1} \sup _{n \leq m}\left\|\mathcal{E}_{n}\left|x_{m}-x_{n-1}\right|^{2}\right\|_{\infty}\right)^{1 / 2} .
$$

Similarly, the row version $\mathrm{BMO}_{\mathrm{R}}(\mathcal{M})$ is defined as the space of all $x$ for which $x^{*} \in \operatorname{BMO}_{\mathrm{C}}(\mathcal{M})$, equipped with the natural norm $\|x\|_{\mathrm{BMO}_{\mathrm{R}}(\mathcal{M})}=$ $\left\|x^{*}\right\|_{\mathrm{BMO}_{\mathrm{C}}(\mathcal{M})}$. The space $\mathrm{BMO}(\mathcal{M})$ associated with the filtration $\left(\mathcal{M}_{n}\right)$ is the intersection of the two types of BMO-spaces described above:

$$
\operatorname{BMO}(\mathcal{M}):=\operatorname{BMO}_{\mathrm{C}}(\mathcal{M}) \cap \mathrm{BMO}_{\mathrm{R}}(\mathcal{M})
$$

with the usual intersection norm of two Banach spaces:

$$
\|x\|_{\mathrm{BMO}}=\max \left\{\|x\|_{\mathrm{BMO}_{\mathrm{C}}},\|x\|_{\mathrm{BMO}_{\mathrm{R}}}\right\} .
$$

As noted above, $\left(\mathcal{H}^{1}(\mathcal{M})\right)^{*}=\operatorname{BMO}(\mathcal{M})$ isomorphically with the isomorphism given by the fact that any $y \in \operatorname{BMO}(\mathcal{M})$ defines a linear functional $\xi_{y}$ on $\mathcal{H}^{1}(\mathcal{M})$ defined by $\xi_{y}(x)=\lim _{n \rightarrow \infty} \operatorname{tr}\left(y_{n}^{*} x_{n}\right)$ for all $x \in \mathcal{H}^{1}(\mathcal{M})$.

For more information on non-commutative martingale BMO-spaces, we refer to the articles $[20,10,15,9]$. Special attention will be given to the subspace called vanishing mean oscillation, denoted by $\operatorname{VMO}(\mathcal{M})$, and defined as the closure (for the BMO-norm) of the linear subspace of those $x \in \operatorname{BMO}(\mathcal{M})$ for which $\mathcal{E}_{n}(x)=x$ for some $n \in \mathbb{N}$.

As in the classical case, for $1 \leq p<\infty, \operatorname{BMO}(\mathcal{M})$ is a subset of $L^{p}(\mathcal{M})$ in the sense of Proposition 2.2 below. These inclusions will be used repeatedly below.

Proposition 2.2 ([9, Theorem 3.18]). For $1 \leq p<\infty$ and $0 \leq \eta \leq 1$, let $I_{p}^{\eta}(x)=D^{(1-\eta) / p} x D^{\eta / p}$. Then the inclusion map

$$
I_{p}^{\eta}(\mathrm{BMO}) \subset L^{p}(\mathcal{M})
$$

is bounded with norm $c(p) \leq c p$, where $c$ is an absolute constant.

3. Main results. Our primary objective in this section is to provide a Kadets-Pełczyński type alternative for VMO-spaces. It generalizes a result of Müller and Schechtman from [14] stated in Theorem 1.1 to non-commutative setting.

For the general statement, we introduce the following norm for $x$ in $\operatorname{BMO}(\mathcal{M})$ :

$$
\|x\|_{\mathrm{C}}:=\sum_{n \geq 1} 2^{-n}\left\|\mathcal{E}_{n}\left(\left|x-\mathcal{E}_{n-1}(x)\right|^{2}\right)\right\|_{\infty}^{1 / 2} .
$$

As above, the row version is defined by $\|x\|_{\mathrm{R}}:=\left\|x^{*}\right\|_{\mathrm{C}}$ and

$$
\|x\|:=\max \left\{\|x\|_{\mathrm{C}},\|x\|_{\mathrm{R}}\right\} .
$$

One can easily verify that $\|\cdot\|$ is a norm in the linear space $\operatorname{BMO}(\mathcal{M})$ with $\|x\| \leq\|x\|_{\mathrm{BMO}}$ for all $x \in \operatorname{BMO}(\mathcal{M})$. We will see below that in general $\|\cdot\|$ 
is not equivalent to the usual BMO-norm. The following property of $\|\cdot\|$ is needed in the proof of our main result.

Lemma 3.1. For every $m_{0} \in \mathbb{N}$ and $z \in \operatorname{VMO}(\mathcal{M})$,

$$
\left\|\mathcal{E}_{m_{0}}(z)\right\|_{\mathrm{VMO}} \leq 2^{m_{0}}\|z\| .
$$

Proof. If $m>m_{0}$, then

$$
\left\|\mathcal{E}_{m}\left|\mathcal{E}_{m_{0}}(z)-\mathcal{E}_{m-1}\left(\mathcal{E}_{m_{0}}(z)\right)\right|^{2}\right\|_{\infty}=\left\|\mathcal{E}_{m}\left|\mathcal{E}_{m_{0}}(z)-\mathcal{E}_{m_{0}}(z)\right|^{2}\right\|_{\infty}=0 .
$$

We deduce from this observation that

$$
\begin{aligned}
\left\|\mathcal{E}_{m_{0}}(z)\right\|_{\mathrm{BMO}_{\mathrm{C}}} & =\sup _{m \leq m_{0}}\left\|\mathcal{E}_{m}\left|\mathcal{E}_{m_{0}}(z)-\mathcal{E}_{m-1}\left(\mathcal{E}_{m_{0}}(z)\right)\right|^{2}\right\|_{\infty}^{1 / 2} \\
& \leq \sup _{m \leq m_{0}}\left\|\mathcal{E}_{m}\left|z-\mathcal{E}_{m-1}(z)\right|^{2}\right\|_{\infty}^{1 / 2} \leq 2^{m_{0}}\|z\|_{\mathrm{C}} .
\end{aligned}
$$

The lemma follows with similar estimate on $\left\|\mathcal{E}_{m_{0}}(z)\right\|_{\mathrm{BMO}_{\mathrm{R}}}$.

Our first result is a characterization of subspaces of VMO containing subspaces isomorphic to $c_{0}$ in terms of the norm $\|\cdot\|$.

Theorem 3.2. Let $X$ be a subspace of $\operatorname{VMO}(\mathcal{M})$. Then either $X$ contains a subspace isomorphic to $c_{0}$ or the norm $\|\cdot\|$ and the usual $\operatorname{VMO}(\mathcal{M})$ norm are equivalent on $X$.

Theorem 3.2 will be deduced from the following result:

Proposition 3.3. (a) Let $\left(p_{k}\right)_{k \geq 1}$ be a strictly increasing sequence of positive integers, $\left(\varepsilon_{k}\right)_{k \geq 1} \subset \mathbb{R}_{+}$satisfying $\sum_{k=1}^{\infty} \varepsilon_{k}=\delta$ and $\left(x_{k}\right)_{k \geq 1} \subset$ $\operatorname{VMO}(\mathcal{M})$ with:

(i) $\mathcal{E}_{p_{k}}\left(x_{k}\right)=x_{k}$ and $\mathcal{E}_{p_{k-1}}\left(x_{k}\right)=0$;

(ii) $\left\|x_{k}\right\|_{\mathrm{VMO}}=1$

(iii) $\left\|\mathcal{E}_{p_{k-1}}\left(S_{\mathrm{C}}^{2}\left(x_{k}\right)\right)\right\|_{\infty} \leq \varepsilon_{k}$.

Then for any finitely non-zero sequence $\left(\alpha_{k}\right)_{k \geq 1} \subset B\left(l^{2}\right)$,

$$
\sup _{k \geq 1}\left\|\alpha_{k}\right\|_{\infty} \leq\left\|\sum_{k \geq 1} \alpha_{k} \otimes x_{k}\right\|_{\mathrm{BMO}_{\mathrm{C}}\left(B\left(l^{2}\right) \bar{\otimes} \mathcal{M}\right)} \leq(1+\delta)^{1 / 2} \sup _{k \geq 1}\left\|\alpha_{k}\right\|_{\infty},
$$

where the BMO-norm is relative to the filtration $\left(B\left(l^{2}\right) \bar{\otimes} \mathcal{M}_{n}\right)_{n \geq 1}$. In particular, $\left(x_{k}\right)_{k \geq 1}$ is a basic sequence that is $(1+\delta)^{1 / 2}$-equivalent to the unit vector basis of $c_{0}$ in $\mathrm{BMO}_{\mathrm{C}}(\mathcal{M})$.

(b) If one replaces condition (iii) by the weaker condition that

$$
\left\|\mathcal{E}_{p_{k-2}}\left(S_{\mathrm{C}}^{2}\left(x_{k}\right)\right)\right\|_{\infty} \leq \varepsilon_{k} \quad \text { for all } k \geq 3,
$$

then the same conclusion still holds with constant $(2+\delta)^{1 / 2}$.

Proof. Let $\left(\alpha_{k}\right)_{k}$ be a finitely non-zero sequence in $B\left(l^{2}\right)$ and set for convenience

$$
a=\sum_{k} \alpha_{k} \otimes x_{k} \in B\left(l^{2}\right) \bar{\otimes} \mathcal{M} .
$$


For $n \geq 1$, let $\mathbb{E}_{n}=\operatorname{Id} \otimes \mathcal{E}_{n}: B\left(l^{2}\right) \bar{\otimes} \mathcal{M} \rightarrow B\left(l^{2}\right) \bar{\otimes} \mathcal{M}_{n}$ be the corresponding conditional expectation. To prove that $\|a\|_{\mathrm{BMO}_{\mathrm{C}}} \leq(1+\delta)^{1 / 2} \sup _{k}\left\|\alpha_{k}\right\|_{\infty}$, we need to estimate $\left\|\mathbb{E}_{m}\left|a-\mathbb{E}_{m-1}(a)\right|^{2}\right\|_{\infty}$ for all $m \geq 1$. To this end, fix $m \in \mathbb{N}$. Then there exists $n \in \mathbb{N}$ so that $p_{n-1}<m \leq p_{n}$.

First, $\mathbb{E}_{m}\left|a-\mathbb{E}_{m-1}(a)\right|^{2}=\mathbb{E}_{m}\left(\sum_{s \geq m}\left|d_{s}(a)\right|^{2}\right)$. Moreover, we observe that for every $s \geq m$,

$$
d_{s}(a)=\sum_{k \geq n} \alpha_{k} \otimes d_{s}\left(x_{k}\right) .
$$

Indeed, if $k<n$ then $p_{k}<p_{n-1}<m \leq s$ so $x_{k} \in \mathcal{M}_{p_{k}} \subset \mathcal{M}_{s-1}$ and therefore $d_{s}\left(x_{k}\right)=0$. On the other hand, if $l, k \geq n$ with $l \neq k$, then

$$
\mathcal{E}_{m}\left(d_{s}\left(x_{l}^{*}\right) d_{s}\left(x_{k}\right)\right)=0 .
$$

To see this, assume that $l>k \geq n$. Then $m \leq p_{k}<p_{l-1}<p_{l}$. By assumption, $x_{k}$ is in $\mathcal{M}_{p_{k}} \subset \mathcal{M}_{p_{l-1}}$ and so also is $d_{s}\left(x_{k}\right)$. We deduce that

$$
\begin{aligned}
\mathcal{E}_{m}\left(d_{s}\left(x_{l}^{*}\right) d_{s}\left(x_{k}\right)\right) & =\mathcal{E}_{m}\left(\mathcal{E}_{p_{l-1}}\left(d_{s}\left(x_{l}^{*}\right)\right) d_{s}\left(x_{k}\right)\right) \\
& =\mathcal{E}_{m}\left(\left[\mathcal{E}_{s} \mathcal{E}_{p_{l-1}}\left(x_{l}^{*}\right)-\mathcal{E}_{s-1} \mathcal{E}_{p_{l-1}}\left(x_{l}^{*}\right)\right] d_{s}\left(x_{k}\right)\right)=0 .
\end{aligned}
$$

Combining (3.3) and (3.4), we have $\left|d_{s}(a)\right|^{2}=\sum_{k \geq n}\left|\alpha_{k}\right|^{2} \otimes\left|d_{s}\left(x_{k}\right)\right|^{2}$. Thus we have the following estimates:

$$
\begin{gathered}
\mathbb{E}_{m}\left|a-\mathbb{E}_{m-1}(a)\right|^{2}=\mathbb{E}_{m}\left(\sum_{s \geq m}\left|d_{s}(a)\right|^{2}\right)=\sum_{s \geq m} \sum_{k \geq n}\left|\alpha_{k}\right|^{2} \otimes \mathcal{E}_{m}\left|d_{s}\left(x_{k}\right)\right|^{2} \\
\leq\left|\alpha_{n}\right|^{2} \otimes \mathcal{E}_{m}\left(\sum_{s \geq m}\left|d_{s}\left(x_{n}\right)\right|^{2}\right)+\sum_{k \geq n+1}\left|\alpha_{k}\right|^{2} \otimes \mathcal{E}_{m}\left(S_{\mathrm{C}}^{2}\left(x_{k}\right)\right) \\
\leq\left|\alpha_{n}\right|^{2} \otimes\left\|x_{n}\right\|_{\mathrm{BMO}_{\mathrm{C}}}^{2} \mathbf{1}+\sum_{k \geq n+1}\left|\alpha_{k}\right|^{2} \otimes \mathcal{E}_{m}\left(S_{\mathrm{C}}^{2}\left(x_{k}\right)\right) .
\end{gathered}
$$

Observe that for $k \geq n+1$, it follows from the choice of $n$ above that $m \leq p_{k-1}$. From condition (iii), we have for every $k \geq n+1$,

$$
\mathcal{E}_{m}\left(S_{\mathrm{C}}^{2}\left(x_{k}\right)\right)=\mathcal{E}_{m}\left(\mathcal{E}_{p_{k-1}}\left(S_{\mathrm{C}}^{2}\left(x_{k}\right)\right)\right) \leq \varepsilon_{k} \mathcal{E}_{m}(\mathbf{1}) .
$$

We conclude that $\left\|\mathbb{E}_{m}\left|a-\mathbb{E}_{m-1}(a)\right|^{2}\right\|_{\infty} \leq\left\|\alpha_{n}\right\|_{\infty}^{2}+\sum_{k \geq n+1}\left\|\alpha_{k}\right\|_{\infty}^{2} \varepsilon_{k}$ and taking supremum over $m$, we see that $\|a\|_{\mathrm{BMO}_{\mathrm{C}}} \leq(1+\delta)^{1 / 2} \sup _{k}\left\|\alpha_{k}\right\|_{\infty}$. Thus the proof of (a) is complete.

For (b), we only need to adjust the last two lines of (3.5) and write

$$
\begin{aligned}
\mathbb{E}_{m}\left|a-\mathbb{E}_{m-1}(a)\right|^{2} \leq & \left|\alpha_{n}\right|^{2} \otimes\left\|x_{n}\right\|_{\mathrm{BMO}_{\mathrm{C}}}^{2} \mathbf{1}+\left|\alpha_{n+1}\right|^{2} \otimes\left\|x_{n+1}\right\|_{\mathrm{BMO}_{\mathrm{C}}}^{2} \mathbf{1} \\
& +\sum_{k \geq n+2}\left|\alpha_{k}\right|^{2} \otimes \mathcal{E}_{m}\left(S_{\mathrm{C}}^{2}\left(x_{k}\right)\right) .
\end{aligned}
$$

As above, if $k \geq n+2$ then $m<p_{k-2}$ and therefore

$$
\mathcal{E}_{m}\left(S_{\mathrm{C}}^{2}\left(x_{k}\right)\right)=\mathcal{E}_{m}\left(\mathcal{E}_{p_{k-2}}\left(S_{\mathrm{C}}^{2}\left(x_{k}\right)\right)\right)
$$


and hence $\left\|\mathcal{E}_{m}\left(S_{\mathrm{C}}^{2}\left(x_{k}\right)\right)\right\|_{\infty} \leq \varepsilon_{k}$. We conclude as above that

$$
\begin{aligned}
\left\|\mathbb{E}_{m}\left|a-\mathbb{E}_{m-1}(a)\right|^{2}\right\|_{\infty} & \leq\left\|\alpha_{n}\right\|_{\infty}^{2}+\left\|\alpha_{n+1}\right\|_{\infty}^{2}+\sum_{k \geq n+1}\left\|\alpha_{k}\right\|_{\infty}^{2} \varepsilon_{k} \\
& \leq(2+\delta) \sup _{k \geq 1}\left\|\alpha_{k}\right\|_{\infty}^{2} .
\end{aligned}
$$

The proof is complete.

Proof of Theorem 3.2. Let $X$ be a subspace of $\operatorname{VMO}(\mathcal{M})$ such that the $\operatorname{BMO}(\mathcal{M})$-norm and $\|\cdot\|$ are not equivalent on $X$. There exists a sequence $\left(z_{n}\right)$ in the unit sphere of $\left(X,\|\cdot\|_{\mathrm{BMO}}\right)$ with $\lim _{n \rightarrow \infty}\left\|z_{n}\right\|=0$. We claim that there exists a subsequence $\left(z_{n_{k}}\right)$ of $\left(z_{n}\right)$ and a sequence $\left(x_{k}\right)$ satisfying the assumptions of Proposition 3.3 and such that $\left\|z_{n_{k}}-x_{k}\right\|<2^{-k}$ for all $k \geq 1$. This is done by induction. First observe that $\lim _{n \rightarrow \infty}\left\|z_{n}\right\|=0$ is equivalent to

$$
\lim _{n \rightarrow \infty} \max \left\{\left\|\mathcal{E}_{m}\left|z_{n}-\mathcal{E}_{m-1}\left(z_{n}\right)\right|^{2}\right\|_{\infty},\left\|\mathcal{E}_{m}\left|z_{n}^{*}-\mathcal{E}_{m-1}\left(z_{n}^{*}\right)\right|^{2}\right\|_{\infty}\right\}=0,
$$

for all $m \in \mathbb{N}$. Furthermore, it follows from Lemma 3.1 that for every $m \in \mathbb{N}$,

$$
\lim _{n \rightarrow \infty}\left\|\mathcal{E}_{m}\left(z_{n}\right)\right\|_{\mathrm{VMO}}=0 .
$$

Let $n_{1}=1$ and $p_{0}=1$. Choose $p_{1}>1$ such that

$$
\left\|z_{1}-\mathcal{E}_{p_{1}}\left(z_{1}\right)\right\|<4^{-1} \text {. }
$$

For such $p_{1}$, we can choose $n_{2}>n_{1}=1$ and $p_{2}>p_{1}$ such that

$$
\begin{gathered}
\sup _{m \leq p_{1}} \max \left\{\left\|\mathcal{E}_{m}\left|z_{n_{2}}-\mathcal{E}_{m-1}\left(z_{n_{2}}\right)\right|^{2}\right\|_{\infty},\left\|\mathcal{E}_{m}\left|z_{n_{2}}^{*}-\mathcal{E}_{m-1}\left(z_{n_{2}}^{*}\right)\right|^{2}\right\|_{\infty}\right\} \leq \varepsilon_{2}, \\
\left\|\mathcal{E}_{p_{1}}\left(z_{n_{2}}\right)\right\|_{\mathrm{VMO}} \leq 4^{-2}
\end{gathered}
$$

and

$$
\left\|z_{n_{2}}-\mathcal{E}_{p_{2}}\left(z_{n_{2}}\right)\right\|_{\mathrm{VMO}} \leq 4^{-2} .
$$

Assume that $n_{k-1}>\cdots>n_{1}$ and $p_{k-1}>\cdots>p_{1}$ have been chosen. One can choose $n_{k}>n_{k-1}$ and $p_{k}>p_{k-1}$ such that

$$
\begin{gathered}
\sup _{m \leq p_{k-1}} \max \left\{\left\|\mathcal{E}_{m}\left|z_{n_{k}}-\mathcal{E}_{m-1}\left(z_{n_{k}}\right)\right|^{2}\right\|_{\infty},\right. \\
\left.\left\|\mathcal{E}_{m}\left|z_{n_{k}}^{*}-\mathcal{E}_{m-1}\left(z_{n_{k}}^{*}\right)\right|^{2}\right\|_{\infty}\right\} \leq \varepsilon_{k}, \\
\left\|\mathcal{E}_{p_{k-1}}\left(z_{n_{k}}\right)\right\|_{\mathrm{VMO}} \leq 4^{-k},
\end{gathered}
$$

and

$$
\left\|z_{n_{k}}-\mathcal{E}_{p_{k}}\left(z_{n_{k}}\right)\right\|_{\mathrm{VMO}} \leq 4^{-k}
$$

For $k \geq 1$, set

$$
x_{k}=\mathcal{E}_{p_{k}}\left(z_{n_{k}}\right)-\mathcal{E}_{p_{k-1}}\left(z_{n_{k}}\right) .
$$

We claim that the sequence $\left(x_{k}\right)$ satisfies the following: 
(i) $\mathcal{E}_{p_{k}}\left(x_{k}\right)=x_{k}$ and $\mathcal{E}_{p_{k-1}}\left(x_{k}\right)=0$;

(ii) $\left\|z_{n_{k}}-x_{k}\right\|_{\mathrm{VMO}} \leq 2^{-k}$;

(iii) $\left\|\mathcal{E}_{p_{k-1}}\left(S_{\mathrm{C}}^{2}\left(x_{k}\right)\right)\right\|_{\infty} \leq \varepsilon_{k}$,

(iv) $\left\|\mathcal{E}_{p_{k-1}}\left(S_{\mathrm{R}}^{2}\left(x_{k}\right)\right)\right\|_{\infty} \leq \varepsilon_{k}$.

The first item is trivial. The second follows from (3.9) and (3.10). In fact, for every $k \geq 1$, we have

$$
\left\|z_{n_{k}}-x_{k}\right\|_{\mathrm{VMO}} \leq\left\|z_{n_{k}}-\mathcal{E}_{p_{k}}\left(z_{n_{k}}\right)\right\|_{\mathrm{VMO}}+\left\|\mathcal{E}_{p_{k-1}}\left(z_{n_{k}}\right)\right\|_{\mathrm{VMO}} \leq 2.4^{-k} \leq 2^{-k} \text {. }
$$

To verify (iii) and (iv), we observe that for every $k \in \mathbb{N}$,

$$
S_{\mathrm{C}}^{2}\left(x_{k}\right)=\sum_{j=p_{k-1}+1}^{p_{k}}\left|d_{j}\left(z_{n_{k}}\right)\right|^{2} .
$$

This leads to the following estimates:

$$
\begin{aligned}
\mathcal{E}_{p_{k-1}}\left(S_{\mathrm{C}}^{2}\left(x_{k}\right)\right) & =\mathcal{E}_{p_{k-1}}\left(\sum_{j=p_{k-1}+1}^{p_{k}}\left|d_{j}\left(z_{n_{k}}\right)\right|^{2}\right) \leq \mathcal{E}_{p_{k-1}}\left(\sum_{j \geq p_{k-1}}\left|d_{j}\left(z_{n_{k}}\right)\right|^{2}\right) \\
& =\mathcal{E}_{p_{k-1}}\left|z_{n_{k}}-\mathcal{E}_{p_{k-1}-1}\left(z_{n_{k}}\right)\right|^{2} .
\end{aligned}
$$

We can deduce from (3.8) that

$$
\left\|\mathcal{E}_{p_{k-1}}\left(S_{\mathrm{C}}^{2}\left(x_{k}\right)\right)\right\|_{\infty} \leq\left\|\mathcal{E}_{p_{k-1}}\left|z_{n_{k}}-\mathcal{E}_{p_{k-1}-1}\left(z_{n_{k}}\right)\right|^{2}\right\|_{\infty} \leq \varepsilon_{k} .
$$

The same argument may be used for the $S_{\mathrm{R}}^{2}\left(x_{k}\right)$ 's. It follows from Proposition 3.3 that the sequence $\left(x_{k}\right)$ is equivalent to the unit vector basis of $c_{0}$ in $\operatorname{VMO}(\mathcal{M})$. We conclude from (ii) that $\left(z_{n_{k}}\right)$ is equivalent to the unit vector basis of $c_{0}$ in $X$.

In general, the two possibilities in Theorem 3.2 are not necessarily mutually exclusive. This is the case when the von Neumannn algebra $\mathcal{M}$ is not hyperfinite. Indeed, assume that the filtration $\left(\mathcal{M}_{n}\right)$ consists of infinitedimensional von Neumannn subalgebras. It is easy to verify that for every $m \in \mathbb{N}, \mathcal{M}_{m}$ (with its usual operator norm) is isomorphic to a subspace of $\operatorname{VMO}(\mathcal{M})$ (using the canonical inclusion). In particular, $\mathcal{M}_{1}$ embeds isometrically into $\operatorname{VMO}(\mathcal{M})$. It is clear that $\|\cdot\|$ and the usual VMO-norm are equivalent on $X=\mathcal{M}_{1}$ but if $\mathcal{M}_{1}$ is infinite-dimensional then it also contains isometric copies of $c_{0}$ and therefore satisfies both possibilities.

For the case of hyperfinite von Neumannn algebras, the dichotomy from Theorem 3.2 turns out to be mutually exclusive. Moreover, it reduces to characterization of Hilbertian subspaces as in Theorem 1.1. To this end, consider the following Hilbertian norm $\|\cdot\|_{2}$ on $\operatorname{BMO}(\mathcal{M})$. For $x \in \operatorname{BMO}(\mathcal{M})$, let

$$
\|x\|_{2}:=\left(\left\|I_{2}^{0}(x)\right\|_{2}^{2}+\left\|I_{2}^{1}(x)\right\|_{2}^{2}\right)^{1 / 2}=\left(\left\|x D^{1 / 2}\right\|_{2}^{2}+\left\|D^{1 / 2} x\right\|_{2}^{2}\right)^{1 / 2}
$$

where $I_{2}^{0}$ and $I_{2}^{1}$ are the inclusion maps described in Proposition 2.2. 
THEOREM 3.4. Assume that $\mathcal{M}$ is hyperfinite and $\left(\mathcal{M}_{n}\right)_{n \geq 1}$ is a filtration consisting of finite-dimensional von Neumannn subalgebras of $\mathcal{M}$. Let $X$ be a subspace of $\operatorname{VMO}(\mathcal{M})$. Then either $X$ is isomorphic to a Hilbert space and complemented in $\mathrm{BMO}(\mathcal{M})$ or $X$ contains a subspace isomorphic to $c_{0}$.

Proof. Let $T_{2}=I_{2}^{0} \oplus I_{2}^{1}: \operatorname{BMO}(\mathcal{M}) \rightarrow L^{2}(\mathcal{M}) \oplus_{2} L^{2}(\mathcal{M})$ be the linear map given by $x \mapsto\left(x D^{1 / 2}, D^{1 / 2} x\right)$ and $S=\left.T_{2}\right|_{X}$.

- If $S$ is an isomorphism then $X$ is isomorphic to the Hilbert space $S(X)$ and if $P: L^{2}(\mathcal{M}) \oplus_{2} L^{2}(\mathcal{M}) \rightarrow S(X)$ is the orthogonal projection then $\Pi:=S^{-1} P T_{2}$ is a projection from $\mathrm{BMO}(\mathcal{M})$ onto $X$.

- If $S$ is not an isomorphism then the $\|\cdot\|_{2^{-n o r m}}$ and the $\operatorname{VMO}(\mathcal{M})$-norm are not equivalent on $X$ in the sense that there exists a sequence $\left(z_{n}\right)_{n \geq 1}$ in the unit sphere of $X$ such that $\lim _{n \rightarrow \infty}\left(\left\|z_{n} D^{1 / 2}\right\|_{2}+\left\|D^{1 / 2} z_{n}\right\|_{2}\right)=0$. We claim that $\lim _{n \rightarrow \infty}\left\|z_{n}\right\|=0$. To this end, we will verify that for every $m \in \mathbb{N}$, $\lim _{n \rightarrow \infty}\left\|\mathcal{E}_{m}\left|z_{n}-\mathcal{E}_{m-1}\left(z_{n}\right)\right|^{2}\right\|_{\infty}=0$ and $\lim _{n \rightarrow \infty}\left\|\mathcal{E}_{m}\left|z_{n}^{*}-\mathcal{E}_{m-1}\left(z_{n}^{*}\right)\right|^{2}\right\|_{\infty}=0$.

Let $m \geq 1$ be fixed. Since conditional expectations are bounded on $L^{2}(\mathcal{M})$, it is clear from the fact that $\mathcal{E}_{m-1}\left(z_{n} D^{1 / 2}\right)=\mathcal{E}_{m-1}\left(z_{n}\right) D^{1 / 2}$ (see for instance the proof of $\left[10\right.$, Proposition 2.3]) that $\lim _{n \rightarrow \infty}\left\|\mathcal{E}_{m-1}\left(z_{n}\right) D^{1 / 2}\right\|_{2}=0$ and therefore

$$
\lim _{n \rightarrow \infty}\left\|\left(z_{n}-\mathcal{E}_{m-1}\left(z_{n}\right)\right) D^{1 / 2}\right\|_{2}=0 .
$$

Thus

$$
\lim _{n \rightarrow \infty}\left\|D^{1 / 2}\left|z_{n}-\mathcal{E}_{m-1}\left(z_{n}\right)\right|^{2} D^{1 / 2}\right\|_{1}=0 .
$$

Since the conditional expectation $\mathcal{E}_{m}$ is bounded in $L^{1}(\mathcal{M})$, it follows that

$$
\lim _{n \rightarrow \infty}\left\|D^{1 / 2} \mathcal{E}_{m}\left|z_{n}-\mathcal{E}_{m-1}\left(z_{n}\right)\right|^{2} D^{1 / 2}\right\|_{1}=0 .
$$

On the other hand, $\left(\mathcal{E}_{m}\left|z_{n}-\mathcal{E}_{m-1}\left(z_{n}\right)\right|^{2}\right)_{n \geq 1}$ is a bounded sequence in the finite-dimensional space $\mathcal{M}_{m}$. Any subsequence of $\left(\mathcal{E}_{m}\left|z_{n}-\mathcal{E}_{m-1}\left(z_{n}\right)\right|^{2}\right)_{n \geq 1}$ has a further subsequence that is convergent in $\mathcal{M}_{m}$ and since the inclusion map $I_{1}^{1 / 2}: \mathcal{M} \rightarrow L^{1}(\mathcal{M})$ is continuous (see Proposition 2.2), it follows from (3.13) that the limit of such convergent subsequence must be zero. This proves that

$$
\lim _{n \rightarrow \infty}\left\|\mathcal{E}_{m}\left|z_{n}-\mathcal{E}_{m-1}\left(z_{n}\right)\right|^{2}\right\|_{\infty}=0
$$

Since $m$ is arbitrary, we deduce that $\lim _{n \rightarrow \infty}\left\|z_{n}\right\|_{\mathrm{C}}=0$. A similar argument can be used for $\left(z_{n}^{*}\right)_{n \geq}$ in order to conclude that $\lim _{n \rightarrow \infty}\left\|z_{n}\right\|_{\mathrm{R}}=0$.

REMARKS 3.5. (a) Theorem 3.4 cannot be extended to the larger space $\mathrm{BMO}(\mathcal{M})$. Indeed, since $\mathrm{BMO}(\mathcal{M})$ is a dual Banach space and contains a subspace isomorphic to $c_{0}$, it necessarily contains a subspace isomorphic to $l^{\infty}$ (see for instance [3, Theorem 10, p. 48]). In particular, it contains a subspace isomorphic to $l^{1}$. Such a subspace clearly fails to satisfy the conclusion of Theorem 3.2. 
(b) One should note that in contrast to the case of VMO, the noncommutative generalization of the classical Kadets-Pełczyński alternatives for non-commutative $L^{p}$-spaces $(2<p<\infty)$, settled in [21], does not require the hyperfinite assumption.

We conclude this section with a quantitative form of Proposition 3.4 for the case of hyperfinite filtration. This version is much closer in spirit to the proof in the commutative case of dyadic-VMO considered in [14]. It also illustrates the involvement of Hilbert space norms.

First we will fix some notations. For $n \geq 1$, let $\gamma_{n}:=\operatorname{dim}\left(\mathcal{M}_{n}\right)=$ $\operatorname{dim}\left(L^{2}\left(\mathcal{M}_{n}\right)\right)$. Inductively, we can construct a sequence $\left(w_{j}\right)_{j=1}^{\infty}$ in $\mathcal{M}$ such that:

(a) $\left(w_{j} D^{1 / 2}\right)_{j=1}^{\infty}$ is an orthonormal basis of $L^{2}(\mathcal{M})$;

(b) for every $n \geq 1,\left(w_{j} D^{1 / 2}\right)_{1 \leq j \leq \gamma_{n}}$ is an orthonormal basis for $L^{2}\left(\mathcal{M}_{n}\right)$. For $n \geq 1$, define

$$
\Phi(n):=\sum_{j=1}^{\gamma_{n}}\left\|w_{j}\right\|_{\infty}^{2}, \quad n \geq 1 .
$$

As an illustration, consider the concrete case of the type- $\mathrm{II}_{1}$ hyperfinite factor $\mathcal{R}$ equipped with its usual trace $\tau$ and its usual filtration $\left(\mathcal{R}_{n}\right)_{n \geq 1}$. Since $\mathcal{R}_{n}$ is the matricial space $\mathbb{M}_{2^{n} \times 2^{n}}$, we have $\gamma_{n}=4^{n}$. Moreover, the orthonormal system can be taken to be the non-commutative Walsh system $\left\{w_{\gamma}\right\}_{\gamma \in \widehat{D}}$ where $\widehat{D}$ denotes the dual group of the dyadic group $D=\prod_{n=1}^{\infty} \mathbb{Z} / 2 \mathbb{Z}$. For a detailed account of the non-commutative Walsh system, we refer to [1]. It was shown in [1] that $\left\{w_{\gamma}\right\}_{\gamma \in \widehat{D}}$ is an orthonormal basis of $L^{2}(\mathcal{R}, \tau)$. Also, $\left\{w_{\gamma}\right\}_{\gamma \in D_{2 n}^{*}}$ where $D_{2 n}^{*}=\left\{\gamma \in \widehat{D}: \gamma_{i}=0\right.$ when $\left.i>n\right\}$ is a basis of $\mathcal{R}_{n}$ and $\left\|w_{\gamma}\right\|_{\infty}=1$ for every $\gamma \in \widehat{D}$. Thus in this case $\Phi(n)=4^{n}$ for every $n \geq 1$.

Proposition 3.6. Let $\left(p_{k}\right)_{k \geq 1}$ be a strictly increasing sequence of integers, $\left(\varepsilon_{k}\right)_{k \geq 1} \subset \mathbb{R}_{+}$satisfying $\sum_{k=1}^{\infty} \varepsilon_{k}=\delta$ and $\left(x_{k}\right)_{k \geq 1} \subset \operatorname{VMO}(\mathcal{M})$ with:

(i) $\mathcal{E}_{p_{k}}\left(x_{k}\right)=x_{k}$ and $\mathcal{E}_{p_{k-1}}\left(x_{k}\right)=0$;

(ii) $\left\|x_{k}\right\|_{\mathrm{VMO}}=1$

(iii) $\left\|x_{k} D^{1 / 2}\right\|_{2}^{2}+\left\|D^{1 / 2} x_{k}\right\|_{2}^{2} \leq \varepsilon_{k} \Phi\left(p_{k-2}\right)^{-1}$ for all $k \geq 3$.

Then for any finitely non-zero sequence $\left(\alpha_{k}\right)_{k \geq 1} \subset B\left(l^{2}\right)$,

$$
\sup _{k \geq 1}\left\|\alpha_{k}\right\|_{\infty} \leq\left\|\sum_{k \geq 1} \alpha_{k} \otimes x_{k}\right\|_{\mathrm{BMO}\left(B\left(l^{2}\right) \bar{\otimes} \mathcal{M}\right)} \leq(2+\delta)^{1 / 2} \sup _{k \geq 1}\left\|\alpha_{k}\right\|_{\infty} .
$$

Proof. It is enough to verify that condition (iii) implies the weaker condition (iii) of Proposition 3.3(b). To this end, we will show that for every $k \geq 3$, we have the estimates $\mathcal{E}_{p_{k-2}}\left(S_{\mathrm{C}}^{2}\left(x_{k}\right)\right) \leq \varepsilon_{k}$ and $\mathcal{E}_{p_{k-2}}\left(S_{\mathrm{R}}^{2}\left(x_{k}\right)\right) \leq \varepsilon_{k}$. 
Let $k \geq 3$ and consider $\mathcal{E}_{p_{k-2}}\left(S_{\mathrm{C}}^{2}\left(x_{k}\right) D^{1 / 2}\right) \in L^{2}\left(\mathcal{M}_{p_{k-2}}\right)$. Since the system $\left(w_{j} D^{1 / 2}\right)_{j \leq \gamma_{p_{k-2}}}$ is an orthonormal basis of $L^{2}\left(\mathcal{M}_{p_{k-2}}\right)$, we have the expansion

$$
\begin{aligned}
\mathcal{E}_{p_{k-2}}\left(S_{\mathrm{C}}^{2}\left(x_{k}\right)\right) D^{1 / 2} & =\sum_{j=1}^{\gamma_{p_{k-2}}}\left\langle\mathcal{E}_{p_{k-2}}\left(S_{\mathrm{C}}^{2}\left(x_{k}\right) D^{1 / 2}\right), w_{j} D^{1 / 2}\right\rangle w_{j} D^{1 / 2} \\
& =\sum_{j=1}^{\gamma_{p_{k-2}}}\left\langle\mathcal{E}_{p_{k-2}}\left(D^{1 / 2} S_{\mathrm{C}}^{2}\left(x_{k}\right) D^{1 / 2}\right), w_{j}\right\rangle w_{j} D^{1 / 2} .
\end{aligned}
$$

Since $D^{1 / 2}$ is fully supported, it follows that

$$
\mathcal{E}_{p_{k-2}}\left(S_{\mathrm{C}}^{2}\left(x_{k}\right)\right)=\sum_{j=1}^{\gamma_{p_{k-2}}}\left\langle\mathcal{E}_{p_{k-2}}\left(D^{1 / 2} S_{\mathrm{C}}^{2}\left(x_{k}\right)\right) D^{1 / 2}, w_{j}\right\rangle w_{j} .
$$

We deduce from the $L^{1}$-boundedness of expectations and the definition of $\Phi$ that

$$
\begin{aligned}
\left\|\mathcal{E}_{p_{k-2}}\left(S_{\mathrm{C}}^{2}\left(x_{k}\right)\right)\right\|_{\infty} & \leq \sum_{j=1}^{\gamma_{p_{k-2}}}\left|\left\langle\mathcal{E}_{p_{k-1}}\left(D^{1 / 2} S_{\mathrm{C}}^{2}\left(x_{k}\right) D^{1 / 2}\right), w_{j}\right\rangle\right| \cdot\left\|w_{j}\right\|_{\infty} \\
& \leq \sum_{j=1}^{\gamma_{p_{k-2}}}\left\|\mathcal{E}_{p_{k-2}}\left(D^{1 / 2} S_{\mathrm{C}}^{2}\left(x_{k}\right) D^{1 / 2}\right)\right\|_{1} \cdot\left\|w_{j}\right\|_{\infty}^{2} \\
& \leq \sum_{j=1}^{\gamma_{p_{k-2}}}\left\|D^{1 / 2} S_{\mathrm{C}}^{2}\left(x_{k}\right) D^{1 / 2}\right\|_{1} \cdot\left\|w_{j}\right\|_{\infty}^{2} \\
& =\left\|D^{1 / 2} S_{\mathrm{C}}^{2}\left(x_{k}\right) D^{1 / 2}\right\|_{1} \Phi\left(p_{k-2}\right) .
\end{aligned}
$$

Since $D^{1 / 2} S_{\mathrm{C}}^{2}\left(x_{k}\right) D^{1 / 2}=\sum_{j=p_{k-1}+1}^{p_{k}}\left|d_{j}\left(x_{k} D^{1 / 2}\right)\right|^{2}=S_{\mathrm{C}}^{2}\left(x_{k} D^{1 / 2}\right)$, we get

$$
\begin{aligned}
\left\|\mathcal{E}_{p_{k-2}}\left(S_{\mathrm{C}}^{2}\left(x_{k}\right)\right)\right\|_{\infty} & \leq\left\|S_{\mathrm{C}}\left(x_{k} D^{1 / 2}\right)\right\|_{2}^{2} \Phi\left(p_{k-2}\right) \\
& =\left\|x_{k} D^{1 / 2}\right\|_{2}^{2} \Phi\left(p_{k-2}\right) \leq \varepsilon_{k} .
\end{aligned}
$$

A similar estimate can be applied to the $S_{\mathrm{R}}^{2}\left(x_{k}\right)$ 's and the conclusion follows directly from Proposition 3.3(b).

4. Banach space properties of VMO. Throughout this section, we assume that the von Neumannn algebra $\mathcal{M}$ is hyperfinite and the filtration consists of finite-dimensional subalgebras. We keep all notation introduced in previous sections. First we recall some Banach space concepts.

Definition 4.1. A formal series $\sum_{n=1}^{\infty} x_{n}$ in a Banach space $E$ is called weakly unconditionally Cauchy (WUC) if $\sum_{n=1}^{\infty}\left|\left\langle x^{*}, x_{n}\right\rangle\right|<\infty$ for all $x^{*} \in E^{*}$. 
It is a well known result of Bessaga and Pełczyński that a basic sequence equivalent to the unit vector basis of $c_{0}$ forms a (WUC) series, and conversely, any basic sequence for which $\inf _{n}\left\|x_{n}\right\|>0$ and $\sum_{n=1}^{\infty} x_{n}$ is a (WUC) series is equivalent to the unit vector basis of $c_{0}$. We refer to [3, Theorem 6, p. 44] for equivalent characterizations of (WUC) series.

Evidently, if $\sum x_{n}$ is a (WUC) series and we set $e_{n}=\sum_{j=1}^{n} x_{j}$ for all $n$, then $\left(e_{n}\right)$ is weakly Cauchy and therefore has a weak*-limit in $E^{* *}$. We now consider a notion introduced by Pełczyński $([18,17])$.

Definition 4.2. A Banach space $E$ has property $(u)$ if for every weakly Cauchy sequence $\left(x_{n}\right)$, there exists a (WUC) series $\sum a_{n}$ in $E$ such that $\left(x_{n}-\sum_{j=1}^{n} a_{j}\right)$ is weakly null.

For example, any Banach space with unconditional basis has property $(u)$ and so also do all weakly sequentially complete Banach spaces ([18]). Other classes of Banach spaces having property $(u)$ are order continuous Banach lattices ([12, Proposition 1.c.2, p. 31] ) and those which are $M$-ideals in their biduals $([6])$.

The next result is the main focus of this section. It may be new even for the classical dyadic martingale VMO.

Theorem 4.3. $\operatorname{VMO}(\mathcal{M})$ has property $(u)$.

To prove this theorem, we need two intermediate results. We assume that the next lemma is well known but we include the proof for completeness.

Lemma 4.4. Let $E$ be a real or complex Banach space.

(i) $A$ bounded subset $C$ of $E$ is relatively weakly compact if and only if for a given sequence $\left(x_{n}\right)$ in $C$, there exists a sequence $\left(y_{n}\right)$ with $y_{n} \in \operatorname{conv}\left\{x_{n}, x_{n+1}, \ldots\right\}$ that converges weakly.

(ii) A bounded sequence $\left(x_{n}\right)$ in $E$ converges weakly to an $x$ in $E$ if and only if for any subsequence $\left(x_{n_{k}}\right)$ of $\left(x_{n}\right)$ there exists a sequence $\left(y_{k}\right)$ with $y_{k} \in \operatorname{conv}\left\{x_{n_{k}}, x_{n_{k+1}}, \ldots\right\}$ that converges weakly to $x$.

Proof. Decomposing any functional in $E^{*}$ into its real and imaginary parts, we may assume that $E$ is a real Banach space. We will only prove the non-trivial implications. For (i), we verify (according to James's theorem) that every non-trivial functional $x^{*} \in E^{*}$ attains its maximum on $\overline{\operatorname{conv}}(C)$. To this end, let $x^{*} \in E^{*} \backslash\{0\}$ and set $\alpha:=\sup \left\{\left\langle x^{*}, x\right\rangle: x \in \overline{\operatorname{conv}}(C)\right\}$. Since $\alpha=\sup \left\{\left\langle x^{*}, x\right\rangle: x \in C\right\}$, there exists a sequence $\left(x_{n}\right)$ in $C$ such that $\alpha=\lim _{n \rightarrow \infty}\left\langle x^{*}, x_{n}\right\rangle$. By assumption, there exists a sequence $\left(y_{n}\right)$ with $y_{n} \in$ $\operatorname{conv}\left\{x_{n}, x_{n+1}, \ldots\right\}$ that converges weakly to $x \in \overline{\operatorname{conv}}(C)$. Since $\left\langle x^{*}, y_{n}\right\rangle \in$ $\operatorname{conv}\left\{\left\langle x^{*}, x_{n}\right\rangle,\left\langle x^{*}, x_{n+1}\right\rangle, \ldots\right\}$, it follows that

$$
\left\langle x^{*}, x\right\rangle=\lim _{n \rightarrow \infty}\left\langle x^{*}, y_{n}\right\rangle=\lim _{n \rightarrow \infty}\left\langle x^{*}, x_{n}\right\rangle=\alpha .
$$

Thus $\overline{\operatorname{conv}}(C)$ is relatively weakly compact, and hence so is $C$. 
To verify (ii), it is enough to observe from (i) that $C:=\left\{x_{n}: n \geq 1\right\}$ is relatively weakly compact and since $x$ is the only possible weak cluster point of $C,\left(x_{n}\right)$ converges weakly to $x$.

The proof of Theorem 4.3 is based on the following observation.

REMARK 4.5. First, we note that for every $x \in \operatorname{BMO}(\mathcal{M})$,

$$
\|x\|_{\mathrm{BMO}_{\mathrm{C}}}=\sup _{m \geq 1} \sup _{n \leq m \leq m \leq k} \sup _{n \leq k \leq m}\left\|\mathcal{E}_{n}\left(\sum_{k=n}^{m}\left|d_{k}(x)\right|^{2}\right)\right\|_{\infty}^{1 / 2} .
$$

A similar expression can be used for the $\mathrm{BMO}_{\mathrm{R}}$-norm. It follows that martingale difference sequences are unconditional in $\operatorname{BMO}(\mathcal{M})$. In particular, for every $x \in \operatorname{BMO}(\mathcal{M}), \sum_{k=1}^{\infty} d_{k}(x)$ is a (WUC)-series in $\operatorname{VMO}(\mathcal{M})$. We thank the referee for pointing out this fact.

Proof of Theorem 4.3. Let $\left(x_{n}\right)_{n}$ be a weakly Cauchy sequence in the space $\operatorname{VMO}(\mathcal{M})$ which is not weakly convergent. Viewed as a bounded sequence in the dual Banach space $\operatorname{BMO}(\mathcal{M}),\left(x_{n}\right)_{n \geq 1}$ is a weak*-convergent sequence in $\mathrm{BMO}(\mathcal{M})$. Let $x \in \operatorname{BMO}(\mathcal{M})$ be the weak*-limit of $\left(x_{n}\right)$ in $\operatorname{BMO}(\mathcal{M})$. By Remark 4.5, $\sum_{k=1}^{\infty} d_{k}(x)$ is a (WUC) series in $\operatorname{VMO}(\mathcal{M})$.

For $n \geq 1$, we set

$$
y_{n}=x_{n}-\sum_{k=1}^{n} d_{k}(x)=x_{n}-\mathcal{E}_{n}(x) .
$$

Thus $\left(y_{n}\right)_{n}$ is a bounded sequence in $\operatorname{VMO}(\mathcal{M})$ that converges to zero for the weak* topology in $\operatorname{BMO}(\mathcal{M})$. We claim that the sequence $\left(y_{n}\right)_{n \geq 1}$ is weakly null in $\operatorname{VMO}(\mathcal{M})$.

To prove this claim, we first observe that $\left(y_{n} D^{1 / 2}\right)_{n}$ and $\left(D^{1 / 2} y_{n}\right)_{n}$ are weakly null sequences in $L^{2}(\mathcal{M})$. Let $\left(u_{n}\right)_{n}$ be an arbitrary subsequence of $\left(y_{n}\right)_{n}$. One can choose a block convex combination of $\left(u_{n}\right)_{n}$ (say $\left.\left(v_{n}\right)_{n}\right)$ that converges to zero for the $\|\cdot\|_{2}$-norm. By Theorem 3.2 (and Proposition 3.3), one can choose a further subsequences of $\left(v_{n}\right)_{n}$ (which we still denote by $\left.\left(v_{n}\right)_{n}\right)$ that is equivalent to the unit vector basis of $c_{0}$. In particular, such block convex combinations are weakly null in $\operatorname{VMO}(\mathcal{M})$. We have just verified that the sequence $\left(y_{n}\right)_{n}$ is such that any of its subsequences has a block convex combination that converges to zero weakly. We can now deduce the claim from Lemma 4.4(ii).

In summary, $\sum_{k \geq 1} d_{k}(x)$ is a (WUC) series in $\operatorname{VMO}(\mathcal{M})$ and the sequence $\left(x_{n}-\sum_{k=1}^{n} \bar{d}_{k}(x)\right)_{n \geq 1}$ is weakly null, thus proving that $\operatorname{VMO}(\mathcal{M})$ has property $(u)$.

An immediate consequence of Theorem 4.3 is that every non-weakly compact operator from $\operatorname{VMO}(\mathcal{M})$ into any arbitrary Banach space must fix isomorphic copies of $c_{0}$. This property is called property $(V)$ and is shared 
by $C^{*}$-algebras in general $([19])$. It is known that for dual Banach spaces, property $(V)$ implies the space being Grothendieck. In particular, it follows from [19] that von Neumannn algebras are Grothendieck spaces, i.e. if $\mathcal{N}$ is an arbitrary von Neumannn algebra then any weak*-convergent sequence of functionals in $\mathcal{N}^{*}$ is automatically weakly convergent. We do not know if this property is shared by the space $\operatorname{BMO}(\mathcal{M})$. In particular, the following motivating question seems to be unresolved:

\section{Problem. Is $\operatorname{BMO}(\mathcal{M})$ a Grothendieck space?}

We note however that since $\mathcal{H}_{\mathrm{C}}^{1}(\mathcal{M})$ is a subspace of $L^{1}\left(\mathcal{M} ; l_{\mathrm{C}}^{2}\right)$ which is in turn a (complemented) subspace of $L^{1}\left(\mathcal{M} \bar{\otimes} B\left(l^{2}\right)\right)$, it follows that $\mathrm{BMO}_{\mathrm{C}}(\mathcal{M})=\left(\mathcal{H}_{\mathrm{C}}^{1}(\mathcal{M})\right)^{*}$ is a quotient of the von Neumannn algebra $\mathcal{M} \bar{\otimes}$ $B\left(l^{2}\right)$. Thus $\mathrm{BMO}_{\mathrm{C}}(\mathcal{M})$ has property $(V)$ and therefore is a Grothendieck space. The same observation can be applied to $\mathrm{BMO}_{\mathrm{R}}(\mathcal{M})$. We do not know if this simple argument can be applied to $\operatorname{BMO}(\mathcal{M})$.

Note (added May 18, 2008). After this paper was submitted, Mikhail Ostrovskii informed me that the Müller-Schechtman dichotomy stated in Theorem 1.1 was also independently obtained by M. Leibov for the case of BMO defined on the unit circle (see "Subspaces of the space VMO", Teor. Funktsiǔ Funktsional. Anal. i Prilozhen. 46 (1986), 51-54 (in Russian); translation in J. Soviet Math. 48 (1990), 536-538). I wish to thank M. Ostrovskii for communicating these references to me.

\section{References}

[1] S. A. Ayupov, S. V. Ferleger, and F. A. Sukochev, Isomorphism between the associative and non-associative $L_{p}$-spaces of type $I I_{1}$ hyperfinite factors, Math. Scand. 78 (1996), 271-285.

[2] D. L. Burkholder and R. F. Gundy, Extrapolation and interpolation of quasi-linear operators on martingales, Acta Math. 124 (1970), 249-304.

[3] J. Diestel, Sequences and Series in Banach Spaces, Grad. Texts in Math. 92, Springer, New York, 1984.

[4] J. L. Doob, Stochastic Processes, Wiley, New York, 1953.

[5] A. M. Garsia, Martingale Inequalities: Seminar Notes on Recent Progress, W. A. Benjamin, Reading, MA, 1973.

[6] G. Godefroy and D. Li, Banach spaces which are $M$-ideals in their bidual have property (u), Ann. Inst. Fourier (Grenoble) 39 (1989), 361-371.

[7] U. Haagerup, $L^{p}$-spaces associated with an arbitrary von Neumann algebra, in: Algèbres d'opérateurs et leurs applications en physique mathématique (Marseille, 1977), CNRS, Paris, 1979, 175-184.

[8] W. B. Johnson, Operators into $L_{p}$ which factor through $l_{p}$, J. London Math. Soc. (2) 14 (1976), 333-339.

[9] M. Junge and M. Musat, A noncommutative version of the John-Nirenberg theorem, Trans. Amer. Math. Soc. 359 (2007), 115-142. 
[10] M. Junge and Q. Xu, Noncommutative Burkholder/Rosenthal inequalities, Ann. Probab. 31 (2003), 948-995.

[11] R. V. Kadison and J. R. Ringrose, Fundamentals of the Theory of Operator Algebras. Vol. I, Pure Appl. Math. 100, Academic Press, New York, 1983.

[12] J. Lindenstrauss and L. Tzafriri, Classical Banach Spaces. II, Springer, Berlin, 1979.

[13] P. F. X. Müller, Isomorphisms between $H^{1}$ Spaces, IMPAN Math. Monogr. 66, Birkhäuser, Basel, 2005.

[14] P. F. X. Müller and G. Schechtman, On complemented subspaces of $H^{1}$ and VMO, in: Geometric Aspects of Functional Analysis (1987-88), Lecture Notes in Math. 1376, Springer, Berlin, 1989, 113-125.

[15] M. Musat, Interpolation between non-commutative $B M O$ and non-commutative $L^{p}$ spaces, J. Funct. Anal. 202 (2003), 195-225.

[16] E. Nelson, Notes on non-commutative integration, ibid. 15 (1974), 103-116.

[17] A. Pełczyński, On Banach spaces on which every unconditionally converging operator is weakly compact, Bull. Acad. Polon. Sci. 10 (1962), 641-648.

[18] - A connection between weakly unconditional convergence and weakly completeness of Banach spaces, ibid. 6 (1958), 251-253.

[19] H. Pfitzner, Weak compactness in the dual of a $C^{*}$-algebra is determined commutatively, Math. Ann. 298 (1994), 349-371.

[20] G. Pisier and Q. Xu, Non-commutative martingale inequalities, Comm. Math. Phys. 189 (1997), 667-698.

[21] Y. Raynaud and Q. Xu, On subspaces of non-commutative $L_{p}$-spaces, J. Funct. Anal. 203 (2003), 149-196.

[22] M. Takesaki, Conditional expectations in von Neumann algebras, J. Funct. Anal. 9 (1972), 306-321.

[23] —, Theory of Operator Algebras. I, Springer, New York, 1979.

[24] M. Terp, $L^{p}$-spaces associated with von Neumann algebras, Notes, Copenhagen Univ., 1981.

[25] F. Weisz, Martingale Hardy Spaces and their Applications in Fourier Analysis, Lecture Notes in Math. 1568, Springer, Berlin, 1994.

[26] Q. Xu, Recent development on non-commutative martingale inequalities, in: Functional Space Theory and its Applications (Wuhan), Research Information Ltd, 2003, 283-314.

Department of Mathematics

Miami University

Oxford, OH 45056, U.S.A.

E-mail: randrin@muohio.edu

Received November 11, 2007

Revised version September 7, 2008 